Research Paper

\title{
Salmonella alters heparanase expression and reduces tumor metastasis
}

\author{
Huan-Min Chiu', Wen- Yi Chiou ${ }^{2,3}$, Wei-Jie $\mathrm{Hsu}^{2}$, Li-Hsien $\mathrm{Wu}^{2}$, Ming-Hui Yang ${ }^{4}$, Yu-Chang Tyan ${ }^{5}$ and \\ Che-Hsin Lee $2,3,6,7,8 \bowtie$ \\ 1. Department of Orthopaedics, Kaohsiung Armed Forces General Hospital, Kaohsiung 80284, Taiwan \\ 2. Department of Biological Sciences, National Sun Yat-sen University, Kaohsiung 80424, Taiwan. \\ 3. Aerosol Science Research Center, National Sun Yat-sen University, Kaohsiung, Taiwan, 80424, Taiwan. \\ 4. Department of Medical Education and Research, Kaohsiung Veterans General Hospital, Kaohsiung, Taiwan. \\ 5. Department of Medical Imaging and Radiological Sciences, Kaohsiung Medical University, Kaohsiung, Taiwan. \\ 6. Department of Medical Research, China Medical University Hospital, China Medical University, Taichung 404, Taiwan. \\ 7. Department of Medical Laboratory Science and Biotechnology, Kaohsiung Medical University, Kaohsiung 80708, Taiwan. \\ 8. Doctoral Degree Program in Marine Biotechnology, National Sun Yat-sen University, Kaohsiung 80424, Taiwan. \\ $\square$ Corresponding author: Dr. Che-Hsin Lee, Department of Biological Sciences, National Sun Yat-sen University, Kaohsiung, Taiwan, 70 Lienhai Rd. Kaohsiung \\ 80424, Taiwan. E-mail: chlee@mail.nsysu.edu.tw.
}

() The author(s). This is an open access article distributed under the terms of the Creative Commons Attribution License (https://creativecommons.org/licenses/by/4.0/). See http:/ /ivyspring.com/terms for full terms and conditions.

Received: 2021.03.10; Accepted: 2021.05.30; Published: 2021.06.11

\begin{abstract}
Salmonella causes salmonellosis, is a facultative anaerobe and is one of the common Gram-negative bacteria. Salmonella has anti-tumor potential and tumor-targeting activity. The heparin sulfate on cell surfaces can be cleaved by heparanase that is an endo- $\beta$-D-glucuronidase. Heparanase can destroy the extracellular matrix and is involved in tumor metastasis and angiogenic activity. Previously, Salmonella was demonstrated to inhibit tumor metastasis. It remains unclear whether Salmonella inhibits metastasis by regulating heparanase. The expression of heparanase in Salmonella-treated tumor cells was found to be decreased. Transwell and wound-healing assays demonstrated the inhibition of cell migration after Salmonella treatment. Salmonella was found to influence the levels of phosphate-protein kinase B (P-AKT) and phosphate-extracellular regulated protein kinases (P-ERK), which are involved in heparanase expression. Salmonella reduced the heparanase expression induced upregulating PERK and PAKT signaling pathways. The mice bearing an experimental metastasis tumor model was used to evaluate the anti-tumor metastatic effects of Salmonella. Compared with the control group, Salmonella significantly reduced the number of metastatic nodules and enhanced survival. The results of our study indicate that Salmonella plays a vital role in the inhibition of tumor metastasis through the downregulation of heparanase.
\end{abstract}

Key words: Salmonella; Heparanase; tumor migration; metastasis

\section{Introduction}

Tumor metastasis causes the death of cancer patients, and the complex physiological responses involved are poorly understood. Several genes and changes in proteins are participated in the process of local invasion of tumor cells [1]. In a wide range of tumors, heparin sulfate can be modified by heparanase. The heparanase in tumors correlates with tumor size, metastasis, and prognosis [1, 2]. The reduced tumor growth and metastasis in mice were observed in heparanase knockdown mice [3]. Moreover, heparanase upregulates the expression of many growth factors that are required for metastasis, including fibroblast growth factors (FGF), vascular endothelial growth factor (VEGF), and matrix metalloproteinases (MMPs) [4-6]. Heparanase is involved in tumor metastasis. Many studies have demonstrated that the prognosis of cancer patients is closely related to heparanase expression [7]. Therefore, knockdown of heparanase expression in the tumor microenvironment may control the growth of tumor cells and even prolong the survival of the cancer patients [8]. The use of bacteria as an 
anti-tumor agent dates back to the end of the $19^{\text {th }}$ century, and different bacteria strains have an anti-tumor effect [9]. Some anaerobes can accumulate target hypoxic/necrotic areas of solid tumors and show significant anti-tumor activity. Salmonella, a facultative anaerobe that is capable of growing in hypoxic region of tumors, has been identified as an anti-tumor agent [10]. As Salmonella survives and replicates under both aerobic and hypoxic conditions, it can target small metastatic lesions and more extensive tumor [11, 12]. Our previous studies showed that Salmonella can colonize small tumor nodules and enhance the survival of lung metastatic tumor mice [13]. Moreover, the expression of MMP-9 in tumors was reduced after Salmonella treatment [8]. In the present study, Salmonella was found to reduce the metastasis via the inhibition of heparanase expression.

\section{Materials and Methods}

\section{Cells, bacteria, plasmids, reagents and animals}

The Dulbecco's Modified Eagle's Medium (DMEM) containing $10 \%$ fetal bovine serum gentamicin $(50 \mu \mathrm{g} / \mathrm{mL})$ was used to maintain Murine melanoma cells (B16F10) and murine breast cancer cells (4T1). A vaccine strain of Salmonella choleraesuis [S. choleraesuis subsp. choleraesuis (Smith) Weldin serovar Dublin (ATCC 15480)] was obtained from Bioresources Collection and Research Center (Hsinchu, Taiwan) [13]. As described above, a constitutively active AKT plasmid were used in this study [14-16]. The resveratrol and 4',6-Diamidino-2Phenylindole (DAPI) a were purchased from Sigma-Aldrich (Sigma Aldrich, St. Louis, MO, USA). The National Laboratory Animal Center of Taiwan provided C57BL/ 6 and BABL/c mice. The Laboratory Animal Care and Use Committee of the National Sun Yat-sen-University approved the experimental protocol (permit number: 10634).

\section{Wound-healing and Transwell Assays}

The wound-healing according to the manufacturer's instructions (IBIDI, Martinsried, Germany). The moving distance was detected after 24 $\mathrm{h}$ by using a microscope. The migration distances of untreated cells were set to $100 \%$ and were compared with cells treated with Salmonella. The cell migration according to the manufacturer's instructions (Transwell cultures (ThermoFisher Scientific, Waltham, MA, USA). Cells were stained with DAPI and counted under a fluorescence microscope [8].

\section{Western Blotting and Transfection}

The protein content was determined by a bicinchoninic acid (BCA) protein assay (Pierce
Biotechnology, Rockford, IL, USA). The protein samples was fractionated by SDS-PAGE. Meanwhile, the hybond-enhanced chemiluminescence nitrocellulose membranes (Pall Life Science, Glen Cove, NY, USA) was used to transfer the protein samples. The membranes were incubated with various antibodies, including heparanase (M-45, Santa Cruz Biotechnology, Santa Cruz, CA, USA), AKT (Santa Cruz Biotechnology), phosphorylation-AKT (Santa Cruz Biotechnology), ERK (Santa Cruz Biotechnology), phosphorylation-ERK (Santa Cruz Biotechnology), and $\beta$-actin (Sigma-Aldrich). The appropriate horseradish-peroxidase-conjugated secondary antibodies were used and enhanced chemiluminescence system (T-Pro Biotechnology, New Taipei City, Taiwan) to detect the proteinantibody complexes [17-19]. Lipofectamine 2000 was used to transfect with the constitutively active AKT plasmids to cells. The cells treated with resveratrol for $2 \mathrm{~h}$. After post-transfection or treatment, cells were or were not treated with Salmonella for $90 \mathrm{~min}$. The cell lysates were then harvested.

\section{Bacterial infection}

Various tumor cells $\left(10^{5} /\right.$ well) were cultured in 6 well-plates overnight. Subsequently, 0, 105, 107, $2 \times 10^{7}$ (for B16F10 and 4T1 cells) colony-forming units (cfu) of Salmonella were added to these cells which were cultured in $1 \mathrm{ml}$ of antibiotic-free medium. Tumor cells were incubated for $1.5 \mathrm{~h}$ at $37^{\circ} \mathrm{C}$. All the cells were washed, replenished with gentamicin (100 $\mu \mathrm{g} / \mathrm{ml}$ )-containing complete medium, and further cultured for $16 \mathrm{~h}$.

\section{Quantitative real-time RT-PCR}

The levels of heparanase mRNA in tumor cells infected with Salmonella (multiplicities of infection, MOI $=1,100,200$ ), or mock-infected were determined by the quantitative real-time RT-PCR. Total cellular RNA was isolated and reversed transcribed into cDNA using standard methods. PCR amplification was carried out in the LightCycler system (Roche, Mannheim, Germany) and data analyzed with LightCycler software 3.3 (Roche). The specific primer pairs used for detecting mouse heparanase and $\beta$-actin were 5' - CGA CCG ACG ACG TGG TAG AC and 5'- GCA ACA GCT CCT GGA AGG G, as well as 5'-TGG AAT CCT GTG GCA TCC ATG AAA C and 5'-TAA AAC GCA GCT CAG TAA CAG TCC G, respectively. The copy number of the heparanase gene in each sample was extrapolated from the corresponding standard curve by the indicated software and normalized with the amount of $\beta$-actin in the same sample. 


\section{Proliferation assay}

Salmonella at various MOIs in serum-free medium treated with cells $\left(10^{6} /\right.$ well $)$ for $1.5 \mathrm{~h}$ Cell Counting Kit-8 (Sigma-Aldrich, St. Louis, MO, USA) was used to detect the number of cells.

\section{Mouse experiments}

The B16F10 $\left(10^{5}\right)$ and 4 T1 cells $\left(10^{5}\right)$ admixed with or without Salmonella $(\mathrm{MOI}=200)$ for $1.5 \mathrm{~h}$ and C57BL/ 6 and BALB/c mice were injected with Salmonella-treated or non-treated-cells via the tail vein on Day 0. Tumor-bearing mice were sacrificed, and the serum and lungs were removed, weighed, and histologically examined on day 20 [13]. The heparanase content was measured by an enzymelinked immunosorbent assay (ELISA) kit (LifeSpan BioSciences, Inc, Seattle, WA, USA). In a parallel experiment, mice were monitored for survival.

\section{Statistical analysis}

We determined differences between groups by using an unpaired, two-tailed Student's t-test. The Kaplan-Meier survival curve and log-rank test to measure a survival analysis. A p value less than 0.05 was considered to be statistically significant.

\section{Results}

\section{Salmonella reduced heparanase expression in vitro}

Mouse breast cancer B16F10 melanoma and 4T1 cells were used to study the anti-migration activity of Salmonella. Figure 1 shows the cell survival of and heparanase expression in tumor cells after treatment with Salmonella at various MOIs. We found those MOIs (1-200) that did not induce cytotoxicity after infection with Salmonella for $90 \mathrm{~min}$ and used the conditions to study the heparanase production after Salmonella treatment (Fig. 1A and B). The expressions of heparanase in 4T1 and B16F10 cells were significantly reduced in tumor cells after infection with Salmonella (MOI=200) (Fig. 1 C and D) $(p<0.05$ for
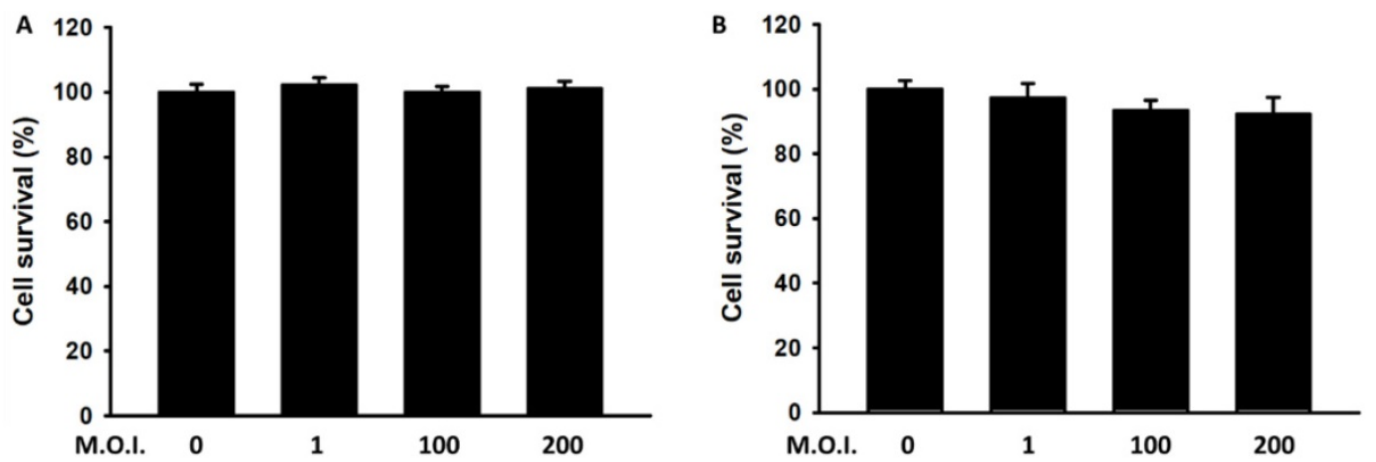

C
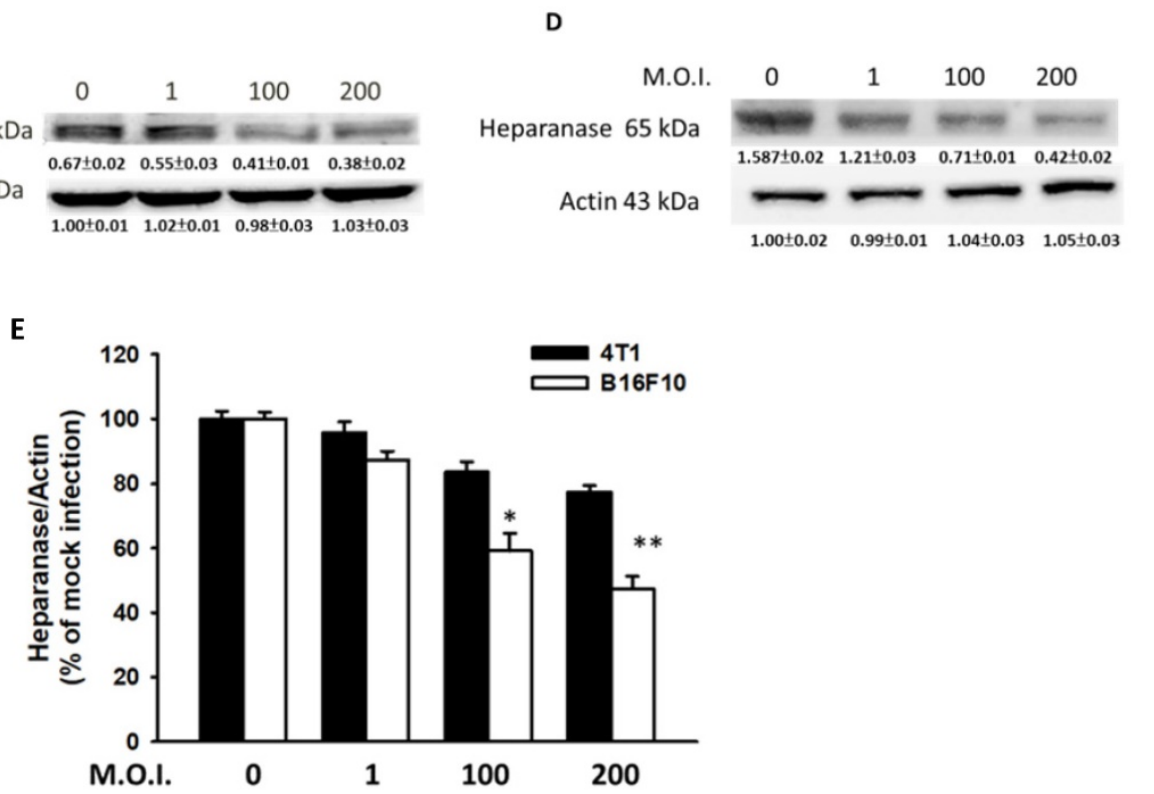

Figure 1. Salmonella (S.C.) regulated cell survival and heparanase protein levels. After infection with Salmonella at various multiplicities of infection (MOls) for $1.5 \mathrm{~h}$, a cell proliferation assay was used in the (A) $4 \mathrm{~T} 1$ and (B) B16F10 cells. $(n=6$, mean \pm SD). The Western blotting was used to detect the protein expression in (C) $4 T 1$ and (D) B16F10 cells. The immunoblotting assay was repeated three times with similar results. Inserted values indicated relative proteins expression in comparison with $\beta$-actin. (E) The real time RT-PCR was used to detect the mRNA expression of heparanase in 4T1 and B16F10 cells. 
S.C. $\mathrm{MOI}=0$ versus $\mathrm{MOI}=200$ in $4 \mathrm{~T} 1 ; \mathrm{p}<0.01$ for S.C. $\mathrm{MOI}=0$ versus $\mathrm{MOI}=200$ in $\mathrm{B} 16 \mathrm{~F} 10$ ). Treatment with an increased amount of Salmonella significantly downregulated the expression of heparanase in the
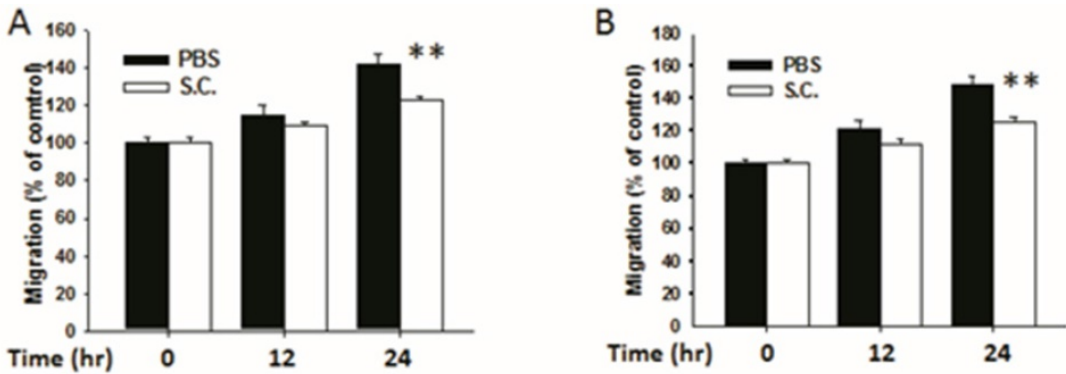

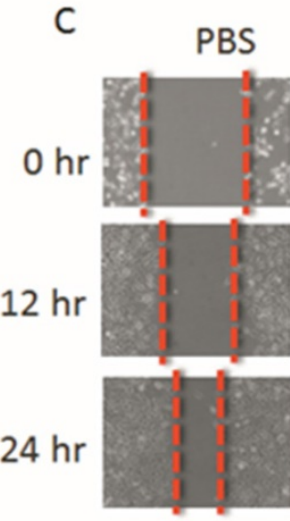

E PBS

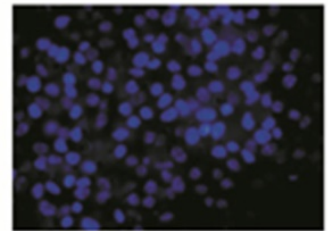

S.C.

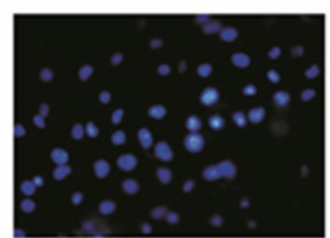

$\mathbf{F}$

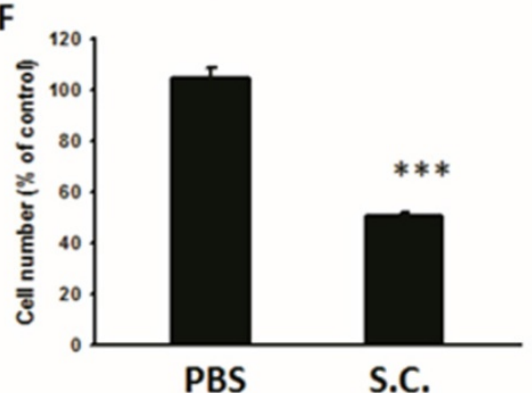

two types of tumor cells. As shown in Fig. 1E, the levels of heparanase mRNA were reduced after Salmonella treatment. Herein, these studies indicate that Salmonella inhibits the protein expression of heparanase in tumor cells.

\section{Salmonella inhibited tumor cell migration}

Salmonella-infected $4 \mathrm{~T} 1$ mouse breast and B16F10 mouse melanoma cells were examined to determine Salmonella's activity to reduce the migration of tumor cells. The results of the wound-healing assay showed that, upon the addition of Salmonella, the movement of 4T1 cells was inhibited compared with that of the control group (Fig. 2A). A similar phenomenon was observed in B16F10 cells infected with Salmonella (Fig. 2B). A woundhealing test was used to observe the reduction of the motility of Salmonella-treated tumor cells (Fig. 2C and D). Although Salmonella did not affect cell proliferation after a short period of infection, there remains the possibility of a reduction in cellular proliferation after Salmonella infection. The results of Transwell assay showed that the migration of 4T1 and B16F10 cells was reduced after treatment with Salmonella (Fig. 2E). After counting the number of migrated tumor cells, the movement of both types of tumor cells was found to be severely affected by Salmonella treatment (Fig. 2F and G). These results suggest that Salmonella reduces the motility of tumor cells.

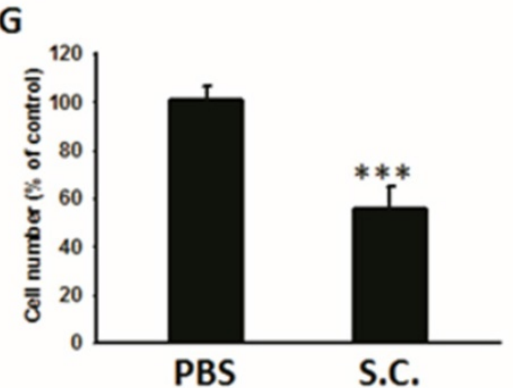

Figure 2. The cellular motility of $4 \mathrm{~T} 1$ and B16F10 cells after Salmonella (S.C.) treatment. The cells were co-cultured with Salmonella $(\mathrm{MOI}=200)$ for $1.5 \mathrm{~h}$. The motility distances of different groups of $(A) 4 T 1$ cells and $(B) B 16 F 10$ cells were measured and are shown in (C, D). The (F) 4T1 cells and $(G) B 16 F 10$ cells were placed on the upper layer of Tranwell and then infected with Salmonella $(\mathrm{MOI}=200)$ for $90 \mathrm{~min}$. After $24 \mathrm{~h}$, the bottom layer of cells were stained with 4',6-diamidino-2-phenylindole (DAPI) and counted under a fluorescence microscope $(E)(n=6$, mean \pm SD. $* *$ $\mathrm{p}<0.01 ; * * * \mathrm{p}<0.001)$.

\section{Salmonella inhibited the} expression of heparanase via the phosphate-protein kinase $B$ (P-AKT) and phosphateextracellular regulated protein kinases (P-ERK) pathways

We used Western blotting to evaluate the potential signaling pathways through which Salmonella has its anti-migration effects and examine the expression of 
heparanase and related signaling pathways (Fig. 3). Heparanase has enzyme activity and induce metastasis. The protein levels of heparanase were inhibited in tumor cells, treated with Salmonella including 4T1 and B16F10 cells (Fig. 3) (p<0.05 for S.C. $\mathrm{MOI}=0$ versus $\mathrm{MOI}=200$ in $4 \mathrm{~T} 1$ and $\mathrm{B} 16 \mathrm{~F} 10)$. This result is consistent with the results shown in Figure 1. Because Salmonella can influence the protein levels of heparanase in two types of tumor cells, Salmonella induced the potential signaling pathway in tumor cells. Some reports have found that AKT and ERK can regulate heparanase synthesis [20]. Previous studies have shown that Salmonella can downregulate phosphate-AKT expression [21]. In this study, treatment with Salmonella was found to reduce the AKT phosphorylation in two tumor cells (Fig. 3) $(\mathrm{p}<0.01$ for S.C. $\mathrm{MOI}=0$ versus $\mathrm{MOI}=200$ in $4 \mathrm{~T} 1$ and B16F10). This effect appears to occur in a dosedependent manner. Phosphate-ERK was also found to be involved in the regulation of cellular heparanase expression [20]. After infection with Salmonella, the expression of phosphate-ERK was significantly inhibited in both cell lines $(p<0.01$ for S.C. MOI=0 versus $\mathrm{MOI}=200$ in $4 \mathrm{~T} 1$ and $\mathrm{B} 16 \mathrm{~F} 10)$. These results suggest that Salmonella inhibits heparanase expression in 4T1 and B16F10 cells through downregulation of the ERK and AKT signaling pathways.

\section{Salmonella reduced heparanase expression via inhibiting the phosphate-AKT and phosphate- ERK signaling pathways}

Phosphate-ERK plays a vital role in Salmonellainduced downregulation of the heparanase pathway. Moreover, resveratrol treatment can upregulate phosphate-ERK [22]. When phosphate-ERK is upregulated after resveratrol treatment, heparanase expression is also increased (Fig. 4A) $(\mathrm{p}<0.01$ for Mock versus resveratrol in 4T1 and B16F10). Salmonella can reduce the expression of heparanase in resveratroltreated cells $(p<0.05$ for S.C. versus resveratrol in 4T1 and B16F10). Furthermore, Salmonella decreased heparanase expression in B16F10 and 4T1 tumor cells by suppressing the phosphorylation of AKT. The transfection of plasmids encoding active form of AKT can rescue the AKT signaling pathway [23]. The suppressive effect of Salmonella on phosphate-AKT was reduced after transfecting the constitutively active form of AKT plasmids in the two types of cells (Fig. 4B) ( $\mathrm{p}<0.05$ for S.C. versus S.C. + AKT in $4 \mathrm{~T} 1$ and B16F10). Transfection of plasmids encoding an active form of AKT increased the expression of heparanase after Salmonella treatment. After transfecting the constitutively active form of AKT, phosphate-ERK was upregulated in the two cell lines $(p<0.05$ for Mock versus AKT in 4T1 and B16F10). Phosphate-AKT was not influenced in the resveratrol-treated group ( $p>0.05$ for AKT versus resveratrol in 4T1 and B16F10). These results suggest that AKT is upstream of ERK in Salmonella-regulated heparanase in 4T1 and B16F10 cells. As previously described, Salmonella significantly reduced the moving distance of tumor cells. The moving distances of Salmonella-treated-4T1 and -B16F10 cells could be reversed by treatment with resveratrol or transfection of a constitutively active AKT plasmid (Fig. 5). The cellular migration behavior was consistent with the results of Western blotting. These results demonstrated that the AKT/ERK signaling pathway might involve in the Salmonellaregulated heparanase expression and tumor cell migration behavior.

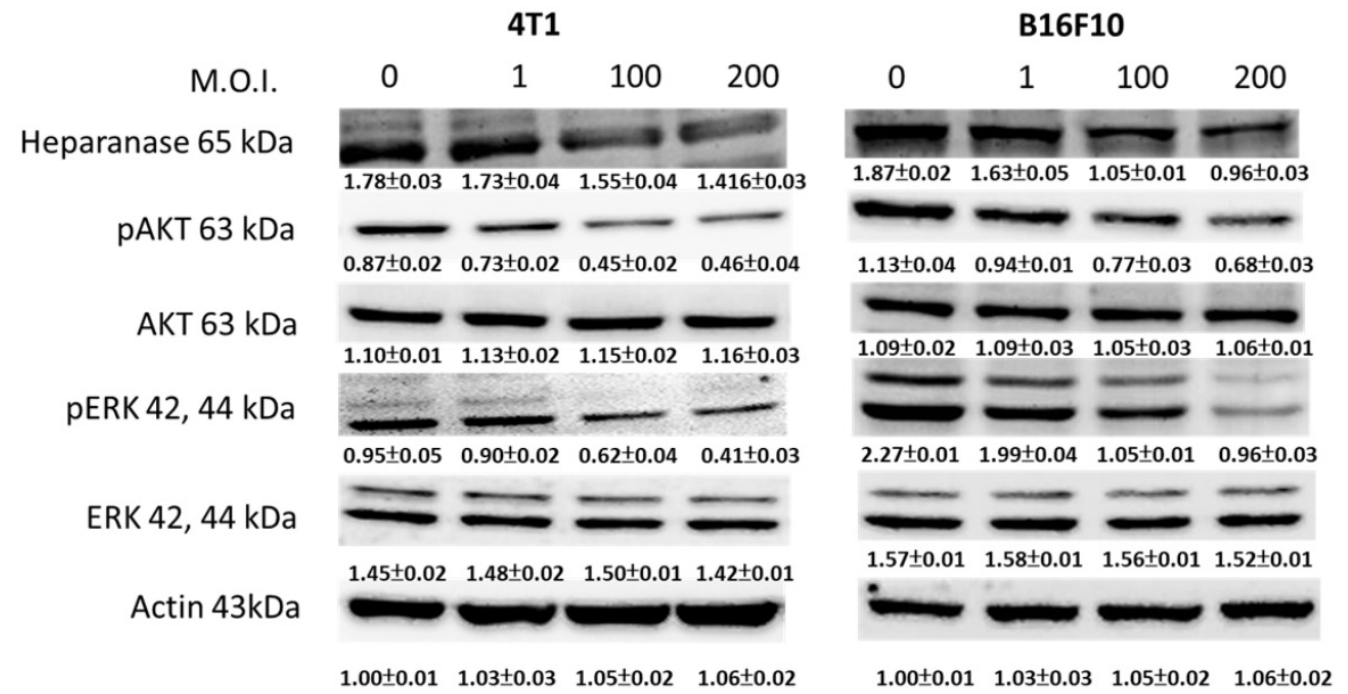

Figure 3. The heparanase expression in Salmonella-treated-4T1 and -B16F10 cells. The cells were co-cultured with Salmonella (MOI = 1-200) for $1.5 \mathrm{~h}$. The protein expression in $4 \mathrm{T1}$ and B16F10 cells was measured. The immunoblotting assay was repeated three times with similar results. Inserted values indicated relative proteins expression in comparison with $\beta$-actin. 


\section{A}

S.C. (M.O.I.=200)
Resveratrol $(4 \mu \mathrm{g} / \mathrm{ml})$
Heparanase $65 \mathrm{kDa}$

pAKT 63 kDa

AKT $63 \mathrm{kDa}$

pERK 42,44 kDa

ERK 42, $44 \mathrm{kDa}$
Actin $43 \mathrm{kDa}$
B $\quad$ S.C. (M.O.I.=200)
AKT
Heparanase $65 \mathrm{kDa}$

pAKT 63 kDa

AKT $63 \mathrm{kDa}$

pERK 42, 44 kDa

ERK 42, $44 \mathrm{kDa}$

Actin $43 \mathrm{kDa}$

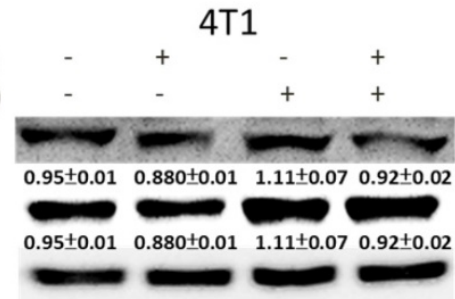

$\begin{array}{llll}1.10 \pm 0.01 & 1.10 \pm 0.03 & 1.09 \pm 0.06 & 1.08 \pm 0.04\end{array}$

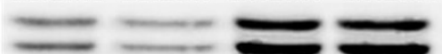

$\begin{array}{llll}1.21 \pm 0.05 & 0.99 \pm 0.02 & 3.20 \pm 0.07 & 3.10 \pm 0.02\end{array}$

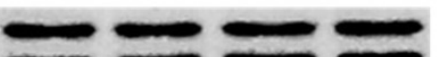

$3.310 \pm 0.053 .39 \pm 0.053 .40 \pm 0.02 \quad 3.42 \pm 0.06$

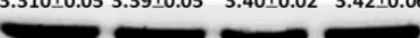

$\begin{array}{llll}1.00 \pm 0.02 & 1.00 \pm 0.03 & 1.01 \pm 0.01 & 1.02 \pm 0.01\end{array}$
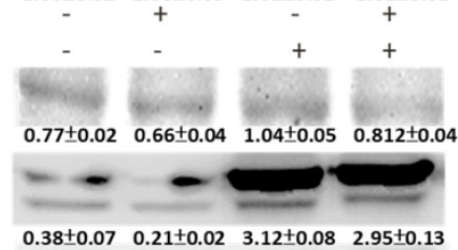

$\begin{array}{llll}0.38 \pm 0.07 & 0.21 \pm 0.02 & 3.12 \pm 0.08 & 2.95 \pm 0.13\end{array}$

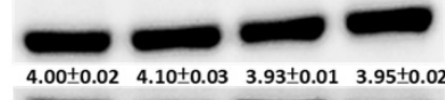

$\begin{array}{llll}4.00 \pm 0.02 & 4.10 \pm 0.03 & 3.93 \pm 0.01 & 3.95 \pm 0.02\end{array}$
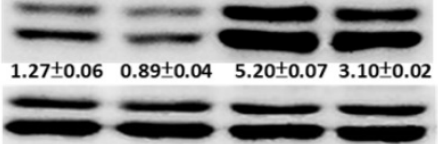

$3.11 \pm 0.043 .13 \pm 0.04 \quad 3.20 \pm 0.06 \quad 3.24 \pm 0.04$

-

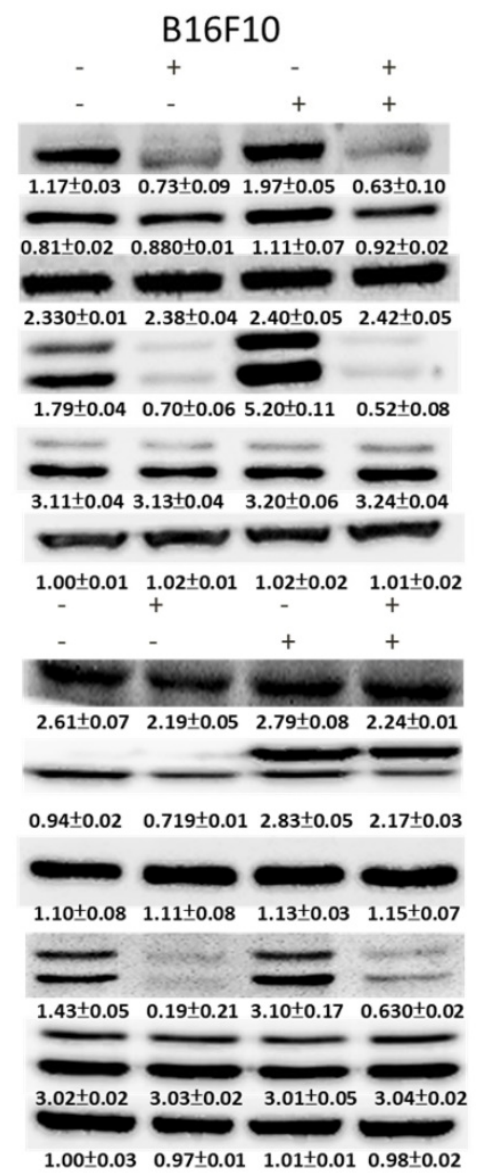

Figure 4. The ERK and AKT signaling pathways were participated in Salmonella (S.C.)-mediated HPSE expression. (A) The 4T1 and B16F10 cells were infected with Salmonella $(\mathrm{MOI}=200)$ at the concentration of $5 \mu \mathrm{g} / \mathrm{mL}$ for $2 \mathrm{~h}$ with resveratrol. The protein expression in $4 \mathrm{~T} 1$ and B16F10 cells was measured. (B) The 4T1 and B16F10 cells were transfected with an active AKT plasmid. The cells were treated with Salmonella $(\mathrm{MOI}=200)$ for $1.5 \mathrm{~h}$ after $16 \mathrm{~h}$. The various protein expressions in $4 \mathrm{~T} 1$ and B16F10 cells was measured. The immunoblotting assay was repeated three times with similar results. Inserted values indicated relative proteins expression in comparison with $\beta$-actin.

\section{Salmonella reduced tumor metastasis in vivo}

The tumor cells can release heparanase and digest extracellular matrixes to help cell migration to distant sites. We previously established a platform that screens for anti-metastatic molecules $[8,24]$. The tumor cells admixed with Salmonella $(\mathrm{MOI}=200)$ and then injected into mice. We investigated whether Salmonella could inhibit pulmonary tumor nodules. Mice bearing metastatic nodules were sacrificed after inoculation of the tumors for 20 days. Serum was collected and analyzed by ELISA. The heparanase in mice treated with Salmonella was significantly decreased in comparison with the PBS group (Fig. 6A). To quantitatively determine tumor burden, the weight of wet lung was measured. The mice injected with 4T1 and B16F10 tumor cells admixed with Salmonella had $63 \%$ and $48 \%$ lower wet lung weight, respectively, compared with those injected with cells admixed with PBS (Fig. 6B). The numerous pulmonary nodules were observed in the lungs from PBS-treated mice. However, the smaller and fewer tumor nodules were observed in the lungs from the mice treated with Salmonella (Fig. 6C). In the two metastatic tumor models, the survival of the mice treated with Salmonella was significantly enhanced (Fig. 6D and E). Collectively, these results show that Salmonella reduced pulmonary tumor nodule growth and prolonged the survival of mice via downregulation of heparanase expression.

\section{Discussion}

Researchers have found Salmonella to possess tumor-targeting potential and considered Salmonella to be new strategies in the treatment of tumors [25, 26]. Salmonella has many tumor-growth-inhibiting qualities, including gene transfer, increasing host immunity, reducing angiogenesis, and inhibiting metastasis $[27,29]$. The mechanism underlying the anti-tumor activity of Salmonella remains vague. Herein, Salmonella reduced the function and expression of heparanase, a major enzyme involved in tumor metastasis, in tumor cells via the AKT/ERK signaling pathway. Salmonella-treated groups had lower heparanase expression, a lower number of metastatic nodules, and lower lung weights in animal models compared with controls. 

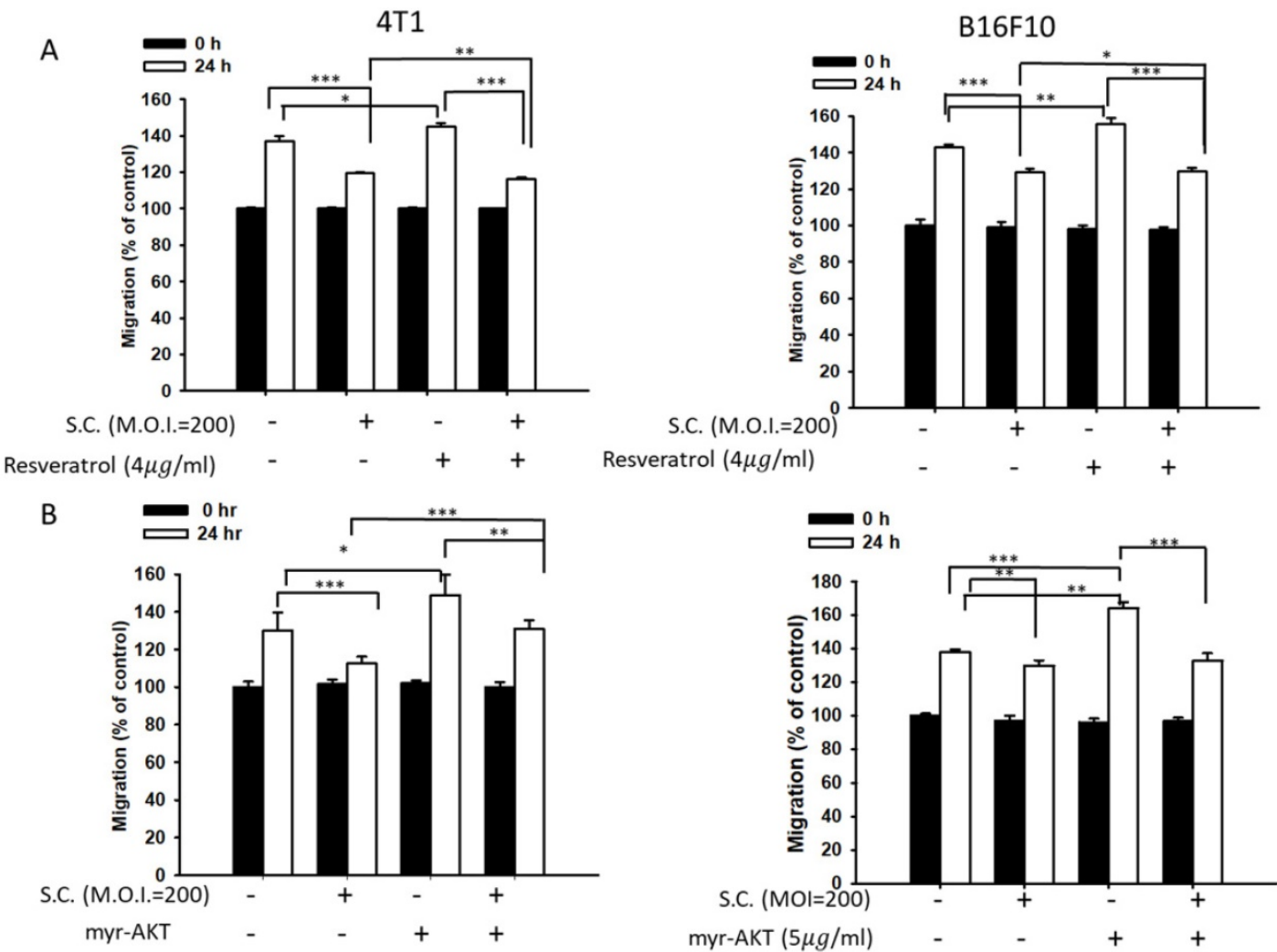

Figure 5. A wound-healing assay showed that the AKT and ERK signaling pathways were participated in the Salmonella (S.C.)-mediated inhibition of tumor cell migration. (A) The $4 \mathrm{Tl}$ and B16F10 cells were infected with Salmonella $(\mathrm{MOI}=200)$ at the concentration of $5 \mu \mathrm{g} / \mathrm{mL}$ for $2 \mathrm{~h}$ with resveratrol. The moving distance of $4 \mathrm{Tl}$ and $\mathrm{B} 16 \mathrm{~F} 10$ cells was measured. (B) The $4 \mathrm{T1}$ and B16F10 cells were transfected with active AKT plasmids. After $16 \mathrm{~h}$, the cells were treated with Salmonella (MOI $=200)$ for $1.5 \mathrm{~h}$. The migration distance of 4 T1 and B16F10 cells was measured $(n=6$, mean \pm SD. $* p<0.05 ; * * p<0.01$; *** $p<0.001)$.
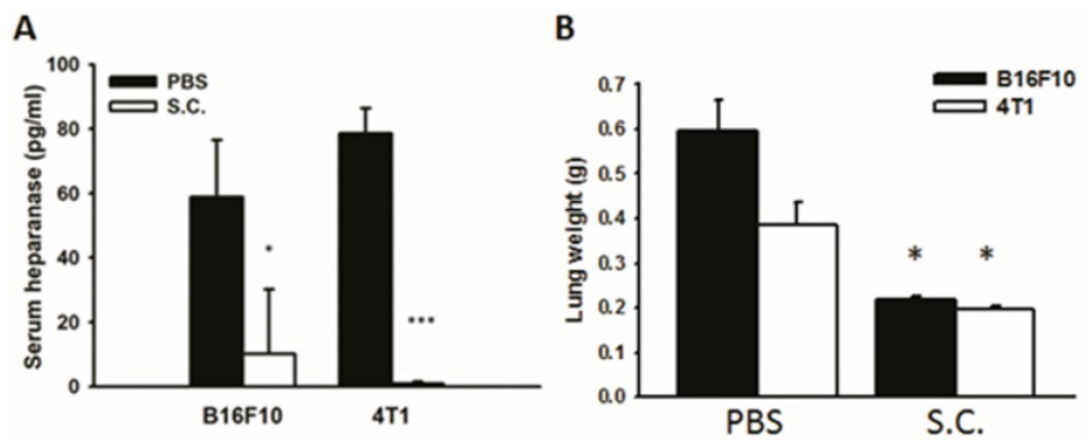

C

C $4 \mathrm{~T} 1$

B16F10

PBS
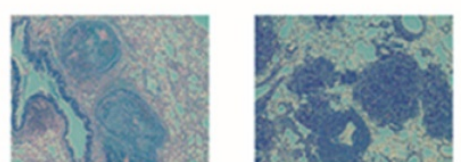

S.C.
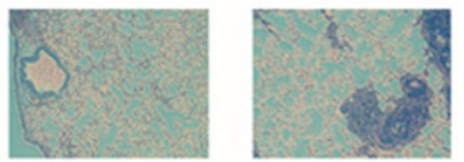

D

$E$
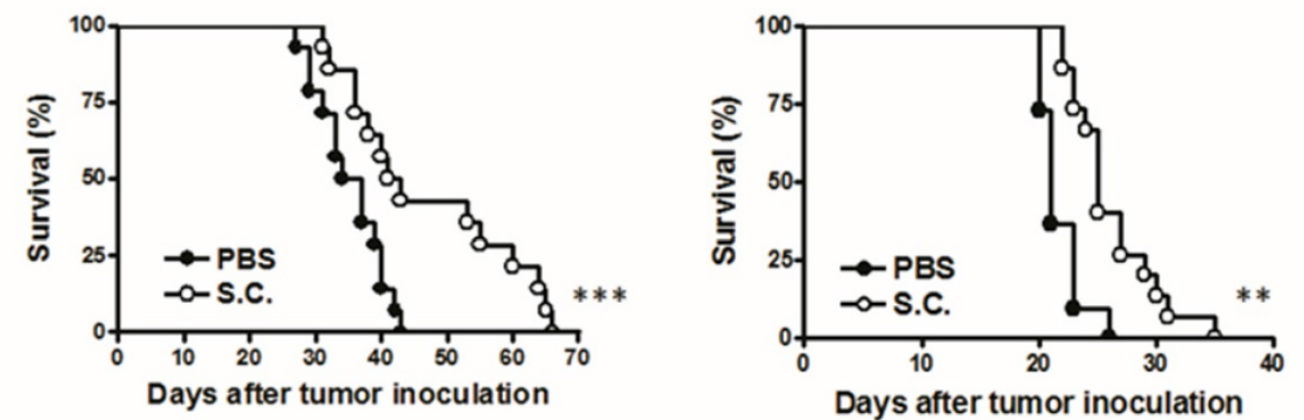

Figure 6. The expression of heparanase was reduced after Salmonella treatment in vivo. Mice were injected with tumor cells $\left(10^{5}\right)$ admixed with or without Salmonella $(\mathrm{MOI}=$ 200 ) for $1.5 \mathrm{~h}$ via the tail vein. At Day 20, the mice were sacrificed. (A) The serum was collected and the protein levels of HPSE were measured. (B) The anti-tumor effect of Salmonella was measured by lung weight $(n=4$, data are expressed as mean $\pm S D . * p<0.05$; $* * *<0.001)$. (C) An example of representative is the picture of metastatic nodules

20 days after intravenous injection of $4 \mathrm{~T} 1$ or B16F10 cells (105). Kaplan-Meier survival curves of mice bearing Salmonella-treated (D) 4T1 and (E) B16F10 tumors are shown (n $=13-15 . * * \mathrm{p}<0.01 ; * * * \mathrm{p}<0.001)$. 
Heparanase not only enhances tumor metastasis but is also involved in the regulation of multiple proteins that promote the aggressive biological behavior of tumor, including VEGF and MMP-9 [3-6]. Previously, we found that Salmonella can reduce tumor migration via reducing MMP-9 [8]. Herein, Salmonella was found to potentially reduce MMP-9 via heparanase [30]. Increasing evidence suggests that heparanase exerts an extreme influence on host immune cells. In transgenic mice, less neutrophil infiltration was observed [31]. When Salmonella accumulates at a tumor site, neutrophils are attracted to tumors [13]. Salmonella-downregulated heparanase expression in tumors may induce neutrophil infiltration. To elucidate the potential mechanism, Salmonella suppressed the expression of AKT/ERK and inhibited heparanase expression. Consistent with the downregulation of heparanase expression, the tumor migration behavior was inhibited. The downregulation of heparanase expression was associated with a decrease in the hypoxia-inducible factor-1a (HIF-1a) level, meaning that the expression of HIF-1a is associated with heparanase [32]. Salmonella has previously been reported to decrease HIF-1a expression [33]. Mechanistically, we found that Salmonella regulated tumor metastasis by downregulating heparanase expression through the AKT/ERK signaling pathway. Heparanase is a multifunctional protein that influenced metastasis and tumor growth. Therefore, heparanase is a viable target for tumor therapy and anti-heparanase is a promising anticancer agent. As a result, several anti-heparanase drugs have been developed to treat tumor [31]. The common side effect associated with anti-heparanase drugs is anti-coagulant activity [34]. Therefore, the ability of Salmonella to target multiple tumors from distant sites makes it an ideal anti-tumor agent over some other cancer therapeutic agents limited to local administration. The anti-heparanse activity was specific in tumor sites by tumor-targeting Salmonella.

Previously, we demonstrated that Salmonella as a single-agent could inhibit tumor growth and enhance survival in mice tumor models [13]. The additive antitumor effects could be observed in the combination therapy of Salmonella plus cisplatin. Our previous findings point out that Salmonella in combination with cisplatin, which exerts oncolytic effects and enhances antitumor immune responses, represents a promising strategy for the treatment of primary and metastatic tumors [13].

The tumor growth rate might be critical factor for Salmonella treatment. The growth rate of B16F10 cells was faster than that of $4 \mathrm{~T} 1$ cells in vitro and in vivo. The rapidly growing $\mathrm{B} 16 \mathrm{~F} 10$ cells are more sensitive to Salmonella-mediated response than the $4 \mathrm{~T} 1$ cells. The AKT/ERK signal pathways in B16F10 cells are more easily influenced after Salmonella treatment compared with $4 \mathrm{~T} 1$ cells (Fig. 4). The growth rate of tumor might be a critical factor involved Salmonella treatment. Furthermore, we have demonstrated that Salmonella can inhibit tumor growth in tumor-bearing mice [14, 17]. We investigate the possible mechanism causing the regression. Salmonella-induced decrease of immune checkpoints can be only seen in vivo but not in vitro. We can verify these findings through analysis of CD4 and CD8 positive cells. However, Salmonella may have pleiotropic activities that can directly and indirectly affect tumor immunity processes in 4T1 tumor models in vivo. As shown in Salmonella-treated 4T1 tumor model, the slightly growth inhibition of 4T1 was observed. The phenomena might be related to Salmonella-mediated the downregulation of immune checkpoints.

Our results demonstrated that downregulating the protein levels of heparanase with Salmonella infection significantly reduced the migration of mouse tumor cells in vitro and in vivo. By analysis of the pleiotropic activities of Salmonella, we suggest that Salmonella not only inhibits primary tumor growth but also reduces metastasis.

\section{Acknowledgements}

This work was supported by grants from the Ministry of Science and Technology, Taiwan (MOST109-2326-B-110-001-MY3), Kaohsiung Armed Forces General Hospital, Taiwan (KAFGH-A-109003), Kaohsiung Veterans General Hospital/National Sun Yat-sen University research project (KSVNSU110013), and Higher Education Sprout Project.

\section{Author Contributions}

H.-M.C. and W.-Y.C. conceived and designed the experiments. W.-Y.C., W.-J.H., L.-H. W. and M.-H. Y. and Y.-C.T. performed the experiments. H.-M.C., W.-Y.C., and C.-H.L. wrote the manuscript.

\section{Competing Interests}

The authors have declared that no competing interest exists.

\section{References}

1. Sanderson RD, Elkin M, Rapraeger AC, Vlodavsky I. Heparanase regulation of cancer, autophagy and inflammation: new mechanisms and targets for therapy. FEBS J. 2017; 284: 42-55.

2. Chen X, Jiang W, Yue C, Zhang W, Tong C, Dai D, Cheng B, Huang C, Lu L. Heparanase contributes to trans-endothelial migration of hepatocellular carcinoma cells. J Cancer. 2017; 8, 3309-3317.

3. Edovitsky E, Elkin M, Zcharia E, Peretz T, Vlodavsky I. Heparanase gene silencing, tumor invasiveness, angiogenesis, and metastasis. J Natl Cancer Inst. 2004; 96: 1219-1230.

4. Zetser A, Bashenko Y, Edovitsky E, Levy-Adam F, Vlodavsky I, Ilan N. Heparanase induces vascular endothelial growth factor expression: correlation 
with p38 phosphorylation levels and Src activation. Cancer Res. 2006; 66: 1455-1463.

5. Nasser NJ, Avivi A, Shafat I, Edovitsky E, Zcharia E, Ilan N, Vlodavsky I, Nevo E. Alternatively spliced Spalax heparanase inhibits extracellular matrix degradation, tumor growth, and metastasis. Proc Natl Acad Sci USA. 2009; 106: $2253-2258$.

6. Cohen-Kaplan V, Naroditsky I, Zetser A, Ilan N, Vlodavsky I, Doweck I. Heparanase induces VEGF C and facilitates tumor lymphangiogenesis. Int J Cancer. 2008; 123: 2566-2573.

7. Purushothaman A, Sanderson RD. Heparanase: A Dynamic Promoter of Myeloma Progression. Adv Exp Med Biol. 2020; 1221: 331-349.

8. Tsao YT, Kuo CY, Cheng SP, Lee CH. Downregulations of AKT/mTOR signaling pathway for Salmonella-mediated suppression of matrix metalloproteinases-9 expression in mouse tumor models. Int J Mol Sci. 2018; 19: pii: E1630.

9. Pawelek JM, Low KB, Bermudes D. Bacteria as tumour-targeting vectors. Lancet Oncol. 2003; 4: 548-556.

10. Pawelek JM, Low KB, Bermudes D. Tumor-targeted Salmonella as a novel anticancer vector. Cancer Res. 1997; 57: 4537-4544.

11. Pangilinan $\mathrm{CR}$, Lee $\mathrm{CH}$. Salmonella-based targeted cancer therapy: updates on a promising and Innovative tumor immunotherapeutic strategy. Biomedicines 2019; 7: pii: E36.

12. Chang WW, Lee $\mathrm{CH}$. Salmonella as an innovative therapeutic antitumor agent. Int J Mol Sci. 2014; 15: 14546-14554.

13. Lee $\mathrm{CH}, \mathrm{Wu}$ C.L, Tai YS, Shiau AL. Systemic administration of attenuated Salmonella choleraesuis in combination with cisplatin for cancer therapy. Mol Ther. 2005; 11: 707-716.

14. Chen MC, Pangilinan CR, Lee CH. Salmonella Breaks Tumor Immune Tolerance by Downregulating Tumor Programmed Death-Ligand 1 Expression. Cancers. 2020; 12(1): pii: E57.

15. Wang $\mathrm{WK}$, Chiang $\mathrm{WC}$, Lai $\mathrm{CH}$, Lee $\mathrm{CH}$ Salmonella-mediated cytolethal distending toxin transfer inhibits tumor growth. Hum Gene Ther. 2018; 29: 1327-1335.

16. Wang CC, Yang CJ, Wu LH, Lin HC, Wen ZH, Lee CH. Eicosapentaenoic acid reduces indoleamine 2,3-dioxygenase 1 expression in tumor cells. Int J Med Sci. 2018; 15: 1296-1303

17. Kuan YD, Lee CH. Salmonella overcomes tumor immune tolerance by inhibition of tumor indoleamine 2, 3-dioxygenase 1 expression. Oncotarget. 2016; 7: 374-385

18. Yang CJ, Kuo CT, Wu LH, Chen MC. Pangilinan CR, Phacharapiyangkul N, Liu W, Chen $\mathrm{YH}$, Lee $\mathrm{CH}$. Eicosapentaenoic acids enhance chemosensitivity through connexin 43 upregulation in murine melanoma models. Int J Med Sci. 2019; 16: 636-643.

19. Chang HL, Kuo YH, Wu LH, Chang CM, Cheng KJ, Tyan YC, Lee CH. The extracts of Astragalus membranaceus overcome tumor immune tolerance by inhibition of tumor programmed cell death protein ligand-1 expression. Int J Med Sci. 2020; 17: 939-945.

20. Spyrou A, Kundu S, Haseeb L, Yu D, Olofsson T, Dredge K, Hammond E, Barash U, Vlodavsky I. Forsberg-Nilsson K. Inhibition of heparanase in pediatric brain tumor cells attenuates their proliferation, invasive capacity, and in vivo tumor growth. Mol Cancer Ther. 2017; 16: 1705-1716.

21. Lee CH, Lin ST, Liu JJ, Chang WW, Hsieh JL. Wang WK. Salmonella induce autophagy in melanoma by the downregulation of AKT/mTOR pathway. Gene Ther. 2014; 21: 309-316.

22. Cheng YJ, Chang MY, Chang WW, Wang WK, Liu CF, Lin ST, Lee CH. Resveratrol enhances chemosensitivity in mouse melanoma model through connexin 43 upregulation. Environ Toxicol. 2015; 30: 877-886.

23. Yang CI, Chang WW, Lin ST, Chen MC, Lee CH. Salmonella overcomes drug resistance in tumor through p-glycoprotein downregulation. Int J Med Sci. 2018; 15: 574-579.

24. Chen MC, Chang WW, Kuan YD, Lin ST, Hsu HC, Lee CH. Resveratrol inhibits LPS-induced epithelial-mesenchymal transition in mouse melanoma model. Innate Immun. 2012; 18: 685-693.

25. Forbes NS. Engineering the perfect (bacterial) cancer therapy. Nat Rev Cancer. 2010; 10: 785-794.

26. Zhao M, Yang M, Li XM, Jiang P, Baranov E, Li S, Xu M, Penman S, Hoffman RM. Tumor-targeting bacterial therapy with amino acid auxotrophs of GFP-expressing Salmonella typhimurium. Proc Natl Acad Sci USA. 2005; 102: 755-760.

27. Hiroshima $Y$, Zhang $Y$, Zhao M, Zhang N, Murakami T, Maawy A, Mii S, Uehara F, Yamamoto M, Miwa S, Yano S, Momiyama M, Mori R, Matsuyama R, Chishima T, Tanaka K, Ichikawa $Y$, Bouvet M, Endo I, Hoffman RM. Tumor-targeting Salmonella typhimurium A1-R in combination with Trastuzumab eradicates HER-2-positive cervical cancer cells in patient-derived mouse models. PLoS One. 2015; 10: e0120358.

28. Yam $\mathrm{C}$, Zhao M, Hayashi $\mathrm{K}, \mathrm{Ma} \mathrm{H}$, Kishimoto $\mathrm{H}$, McElroy M, Bouvet $\mathrm{M}$, Hoffman RM. Monotherapy with a tumor-targeting mutant of $S$. typhimurium inhibits liver metastasis in a mouse model of pancreatic cancer. J Surg Res. 2010; 164: 248-255.

29. Lee $\mathrm{CH}$. Engineering bacteria toward tumor targeting for cancer treatment: current state and perspectives. Appl Microbiol Biotechnol 2012; 93: 517-523.

30. Purushothaman A, Chen L, Yang Y, Sanderson RD. Heparanase stimulation of protease expression implicates it as a master regulator of the aggressive tumor phenotype in myeloma. J Biol Chem. 2008; 283:32628-36.
31. Escobar Galvis ML, Jia J, Zhang X, Jastrebova N, Spillmann D, Gottfridsson E, van Kuppevelt TH, Zcharia E, Vlodavsky I, Lindahl U, Li JP. Transgenic or tumor-induced expression of heparanase upregulates sulfation of heparan sulfate. Nat Chem Biol. 2007; 3: 773-778.

32. Z CZ, Luo C, Yang Z, Wang L. Heparanase participates in the growth and invasion of human U-2OS osteosarcoma cells and its close relationship with hypoxia-inducible factor-1a in osteosarcoma. Neoplasma. 2010; 57: 562-571.

33. Tu DG, Chang WW, Lin ST, Kuo CY, Tsao YT, Lee CH. Salmonella inhibits tumor angiogenesis by downregulation of vascular endothelial growth factor. Oncotarget. 2016; 7: 37513-37523.

34. Groult $\mathrm{H}$, Cousin R, Chot-Plassot $\mathrm{C}$, Maura M, Bridiau N, Piot JM, Maugard T, Fruitier-Arnaudin I. $\lambda$-Carrageenan oligosaccharides of distinct antiheparanase and anticoagulant activities inhibit MDA-MB-231 breast cancer cell migration. Mar Drugs. 2019; 17: pii: E140. 